\title{
Unusual foreign body in the sigmoid colon, chronic alcohol abuse, and Fournier gangrene: a case report
}

\author{
Dietmar Schulz' \\ Georgiana Simona Mohor ${ }^{2}$ \\ Caius Solovan ${ }^{2,3}$ \\ 'Sana Kliniken Leipziger Land, Klinik \\ fur Urologie, Borna, Leipzig, Germany; \\ ${ }^{2}$ University Clinic of Dermatology \\ and Venereology, Timișoara, Romania; \\ ${ }^{3}$ Department of Dermatology, Victor \\ Babeș University of Medicine and \\ Pharmacy, Timișoara, Romania
}

Correspondence: Georgiana Simona Mohor

University Clinic of Dermatology and Venereology, Marasesti nr 5, Timișoara, Romania

Tel +40746086846

Email simona.mohor@gmx.de
This article was published in the following Dove Press journal:

Clinical Interventions in Aging

3I March 2015

Number of times this article has been viewed

\begin{abstract}
Fournier gangrene (FG) is an infectious condition with fulminant evolution and is sometimes life-threatening. Here, we present the case of an immunocompromised 59-year-old male with surgical history of a pancreatic pseudocyst stented endoscopically. After unrecognized stent migration in the sigmoid without colonic perforation, he developed severe necrosis of the scrotum and perineum, which spontaneously perforated, presenting a smell suggesting moist gangrene. FG that has spread to the male genital organs presents therapeutic challenges. The purpose of our study is to present this case, typical for FG, with an educational aim both for the internal and surgical specialties, and the goal of further multidisciplinary collaboration for
\end{abstract} the optimal management of the patient with personalized treatment.

Keywords: pancreatic stent, wound debridement, vacuum-assisted closure, transplanted skin, necrotizing infection

\section{Introduction}

Fournier gangrene (FG) is an infectious condition with fulminant evolution and is sometimes life-threatening. ${ }^{1} \mathrm{FG}$ has a mortality rate of $20 \%-60 \%$ even with treatment and is an acute surgery emergency. ${ }^{2}$ Predisposing factors for FG are considered to be diabetes mellitus, trauma, paraphimosis, extravasation of urine, perirectal or perianal infections, herniorrhaphy, or circumcision. ${ }^{3}$ While preexisting immune suppression of various conditions is observed, the disease also affects healthy individuals. Clinical onset is often insidious, with minimal cutaneous lesions, but typically progresses along deep fascial planes into a rapidly spreading sepsis with a potential fatal outcome in $3 \%-45 \%$ of cases. ${ }^{4-6}$ Because the infection spreads rapidly, treatment measures represent a challenge for wound management. ${ }^{7}$ The main treatment procedures include aggressive hemodynamic stabilization, antibiotic therapy covering all suspected involved bacteria, and emergency surgical debridement. ${ }^{1}$

\section{Case report}

A 59-year-old patient presented himself in August 2013 at the Clinic of Urology Helios Leipziger Land with decreased general well-being, somnolent, cachexia, arterial hypotension, heart rate of $90 \mathrm{bpm}$, respiratory frequency of 25 per minute, and urinary incontinence. Upon clinical tegument examination, severe necrosis of the scrotum and perineum were found (Figure 1). The necrosis had spontaneously perforated, presenting a smell suggesting moist gangrene (Figure 2). Laboratory parameters suggested an inflammatory syndrome (hemoglobin $[\mathrm{mmd} / \mathrm{L}]: 6.7 ; 5.3 ; 5.5 ; 4.8 ; 5.1$; leukocytes [Gpt/L]: 9.8; 9.5; 22.5; 11; 10.6; fibrinogen [g/L]: 8.7; >9; 7.7; 6.3; 4.5; 6; C-reactive 


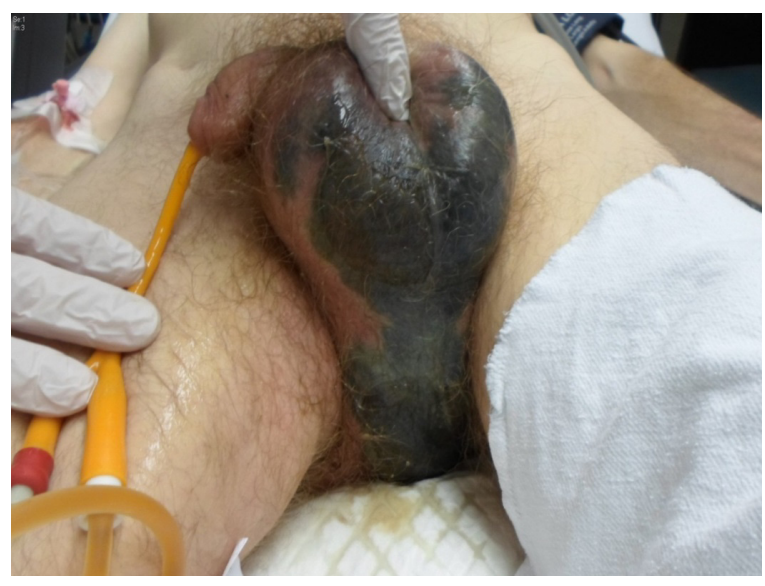

Figure I Severe necrosis of patient's scrotum and perineum.

protein $[\mathrm{mg} / \mathrm{L}]: 327 ; 298 ; 87$; procalcitonin $[\mu \mathrm{g} / \mathrm{L}]: 7.24$; $4.62 ; 2.2 ; 0.51 ; 0.34$; partial thromboplastin time [seconds]: 42.3), and the microbiologic tests indicated a colonization of Bacteroides pyogenes, Staphylococcus haemolyticus, Escherichia coli, Clostridium innocuum, Enterococcus faecium, Enterococcus faecalis, Candida albicans, and non-albicans Candida. Enterococcus faecium was sensitive for macrolidelincosamide-streptogramin, glycylcycline, and the glycopeptide class of antibiotics and resistant to beta-lactam antibiotic, aminoglycoside antibiotic, and the fluoroquinolone drug class. At the clinical general examination, an injury of the right lumbar plexus was observed. Based on clinical appearance and laboratory results, a diagnosis of FG was established.

The patient's pathologic history presented a calcified, chronic pancreatitis as well as a pancreatic pseudocyst into which two stents were applied in order to guide its content into the stomach. During evaluation, however, only one stent was found. This suggested that the other stent might have dislocated and subsequently been eliminated (the other

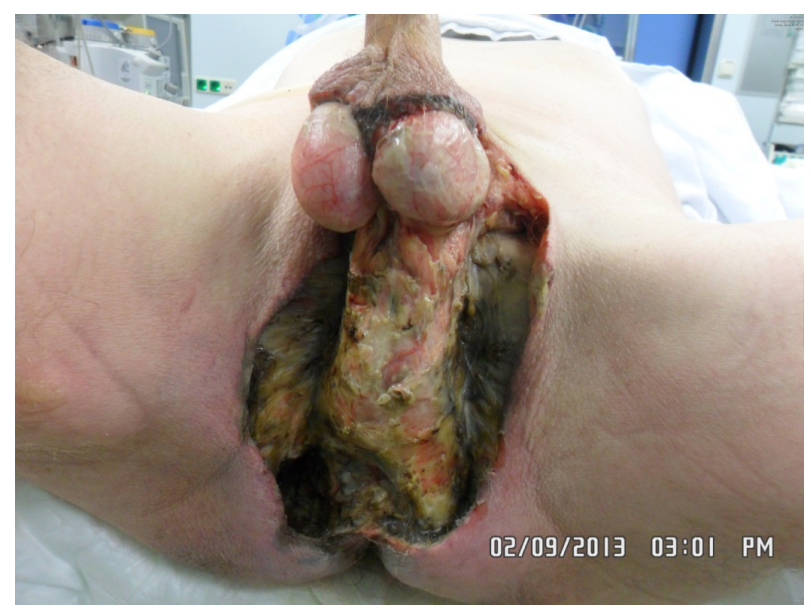

Figure $\mathbf{2}$ The perforation of the necrosis with a smell of moisture gangrene. stent was replaced by an inflatable balloon). After 5 years, upon X-ray computed tomography (CT) examination in the urology clinic, however, a foreign structure of a length of $\sim 10 \mathrm{~cm}$ was observed (his former stent from 2008) in the sigmoid colon, with double perforation and air in the peritoneal cavity as well as massive inflammation of the perivisceral structures. For stent extraction (Figure 3), a sigmoidoscopy was performed, followed by colostomy, sigmoidal anus praeter, and debridement of the scrotum and peritoneum (Figure 4); during surgery, the distal and sigmoid colon were rinsed with saline and no evidence of perforation was further noticed. Surgical necrosis removal and repeated debridement (Figure 5) under antimicrobiotic protection followed. Upon intervention, the patient was transferred from the intensive care unit to the urologic ward. Here, debridement, suprapubic catheter change, and subcutaneous testicle relocation towards the inguinal region continued (Figure 6). The patient was then released with no sign of local infection and recommendation for plastic surgery for visible defect removal was given.

During the subsequent plastic surgery session, the scrotal and periscrotal areas were covered by skin transplantation, the right testicle was repositioned into the inguinal area, and vacuum therapy was applied. Due to rejection and necrosis of the transplanted skin in the middle and left part of the peritoneum, however, the necrotic areas needed to be removed. Vacuum therapy was continued and upon patient request, he was released from the hospital; the wound showed a granular phenotype and it was recommended to have the patches changed on a daily basis.

Due to an ileus induced by adhesion pathology, the patient had a laparotomy with adhesiolysis. From this surgical department, he again left the hospital in good mental health, was spatial and time oriented and cooperative, with

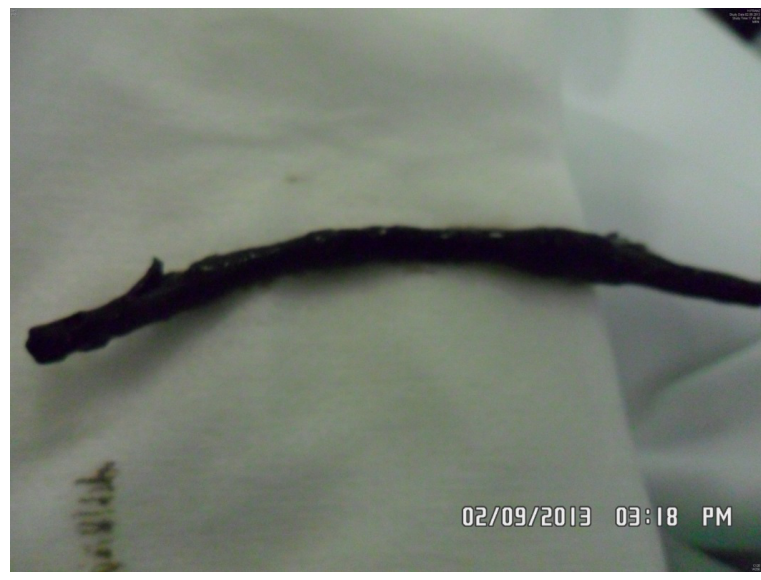

Figure 3 The dislocated stent from 2008, used to guide the pseudo-cyst's content into the stomach. 


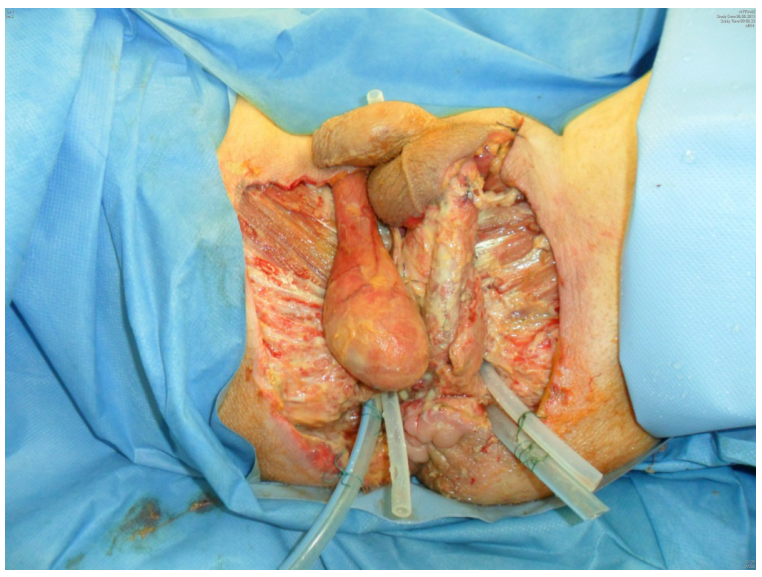

Figure 4 The wound care: debridement - shown is the 8th day post-surgery.

stable blood pressure and sustained, spontaneous breathing frequency; the wound did not show any visible necrotic tissue (Figure 7).

In January 2014, the patient returned to the Department of Urology for a control examination. The operated areas showed no indications of inflammation; the edges were smooth and easily movable. The wounds were dry and well covered on the right side; on the left side, a smooth, pink, shiny area with clean edges was found. Both testicles were slightly smaller, located on the right in the area of the upper femur and on the left in the palpable inguinal area. The scrotal skin was slightly raised below the penoscrotal angle, the preputium was tight, and balanitis had developed. The investigation was described as painful; arbitrary anal sphincter contractions were not possible. The patient was recommended to attend another stationary intervention, more specifically vacuum patch therapy, digital cleaning of the ampulla recti, and check up on the micturition features, followed by balanitis therapy and circumcision, if required.

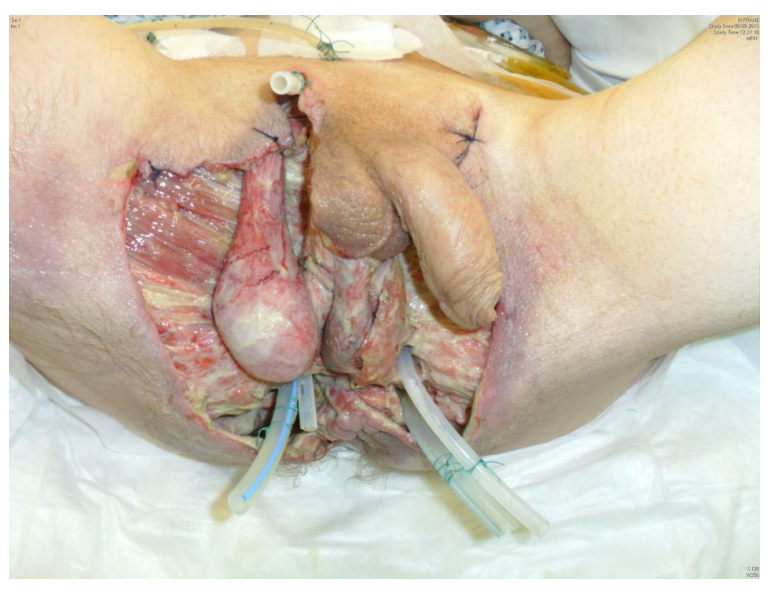

Figure $\mathbf{5}$ The wound care: debridement - shown is the II th day post-surgery.

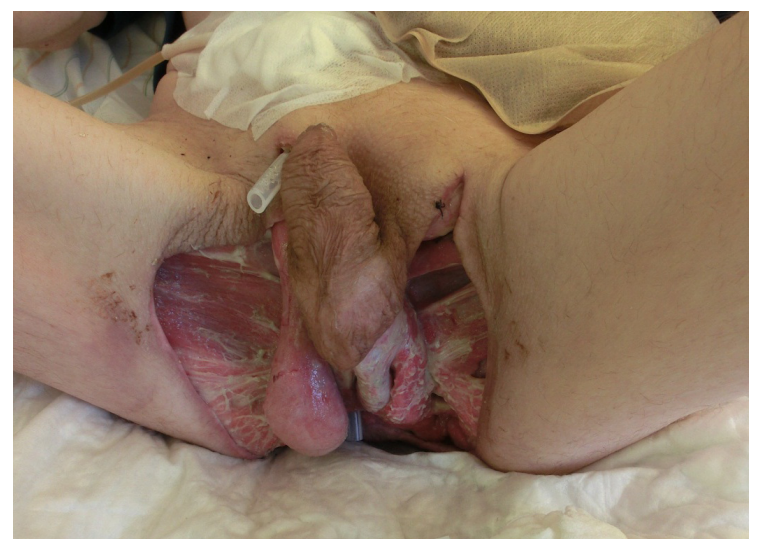

Figure 6 Debridement, suprapubian catheter change and subcutaneous testicle relocation towards the inguinal region - shown is the 30th day post-surgery.

Had the patient preferred to continue with ambulant therapy, the suprapubic catheter would have been closed off in order to induce spontaneous micturition via the urethra.

\section{Discussion}

Due to the tegument appearance of the scrotum with black and yellow-green necrosis, which spontaneously perforated and the specific smell of moist gangrene on this immunocompromised, alcoholic patient, the diagnosis of Fournier necrotizing fasciitis was established. It was also considered that the peripheral necrosis might have appeared due to a septic embolism; even so, septic embolisms normally lead to acral necrosis, with subsequent lower extremity amputation. It is also a well-known fact that the clinical tableau for septic embolism is different from the one typical for Fournier necrotizing fasciitis; the skin is pale and marbled, and the patient is in shock and exhibits acral necrosis, without rotten odor.

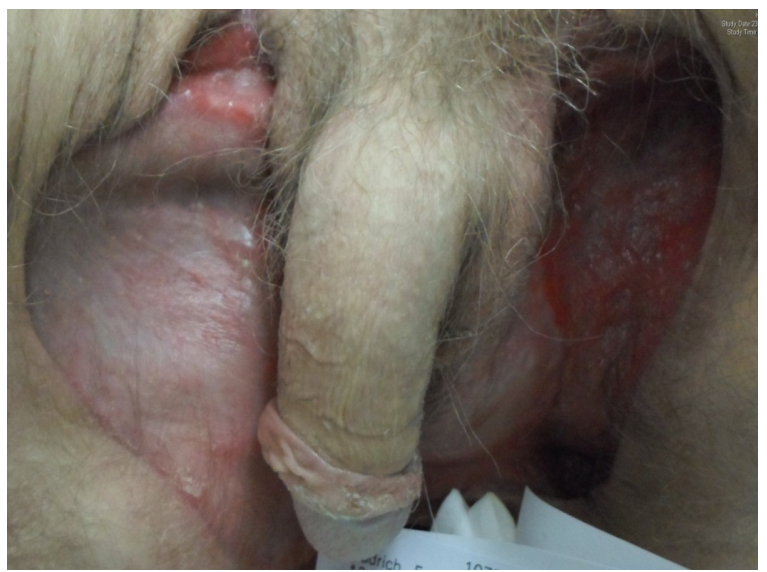

Figure 7 The situation close to patient's release: the wound shows granular tissue formation and is without any visible necrotic areas. 
Another considerable diagnosis could also have been myositis caused by $\beta$-hemolytic group A streptococcus. In contrast to Fournier necrotizing fasciitis, however, for which the central symptom is localized pain, streptococcal myositis is marked by more widespread pain in the extremities. This could easily, but incorrectly, be interpreted as a myalgia; muscle edema occurs later for myalgia and the skin is cold without obvious erythema. The therapeutic approach in streptococcal myositis is early antibiotic application and radical debridement of the lesion, or even leg amputation. ${ }^{8}$ In FG, there are a broad spectrum of antibiotics that could be applied, but infection resistance to these antibiotic classes may occur, as presented in our case.

The dislocated stent could also be considered as a triggering factor. The stent did not lead to any perforation of the bowel wall (which was discovered during surgery) in comparison to the case presented by Marsman and Hoedemaker, where a biliary stent migrated and perforated the sigmoid colon. In our case, the patient's immunodeficiency favored bacterial colonization and infection. ${ }^{9}$

According to the literature, FG affects patients aged 53-58 years old (as per Yilmazlar et al the range is $22-88$ years old). ${ }^{10}$ Recent publications assert that FG tends to appear in increasingly older patients. ${ }^{11}$ The patient from this case report conforms this age tendency; thus, his immunocompromised status precipitated the evolution and influenced the treatment response.

The laboratory risk indicator for necrotizing fasciitis (LRINEC) score is a weighted point system of such markers often used to stratify patients into low, moderate, or high risk for necrotizing soft tissue infection (NSTI). ${ }^{1}$ In our case, the LRINEC score was $>8$, meaning that there indeed was an increased risk for NSTI. FG has a high mortality rate, so urgent diagnosis and treatment is required. This way, the debridement was performed under antibiotic protection. Instead of permanent debridements, another promising variant is the utilization of maggots, which exclusively live on necrotic material. However, the cost of this treatment is higher and expected compliance by patients as well by medical staff can be regarded as relatively limited. The fact that the defect was not covered with a muscle flap (gracilis or rectus abdominis) has shown that the right decision was made. If the muscle flap would not have adhered to the underlying tissue as happened to the skin graft, the patient may have had a much larger supplementary problem. With regard to the vacuum-assisted closure (VAC) that was used on our patient, Pastore et al showed that the utilization of hyperbaric oxygen therapy (HBO) in combination with VAC treatment is effective and that the patient did not require reconstructive surgery. ${ }^{12}$ Hyperbaric oxygen therapy inhibits the development of anaerobic bacteria and reduces the extension of tissue necrosis. ${ }^{13}$ Despite the beneficial action of HBO favoring neutrophilic phagocytic function, angiogenesis, fibroblast proliferation, and intracellular transportation of antibiotics (eg, aminoglycosides), ${ }^{14}$ Grabe et al concluded that the effect of HBO is questionable. ${ }^{15}$ It is likely that the main action of this treatment is against the anaerobic bacteria (especially if Clostridium perfringens is present). Nevertheless, the efficiency of HBO in the treatment of necrotizing fasciitis has not been demonstrated. ${ }^{16}$ In addition to the specific therapy, it is also important to directly treat any comorbidities (alcoholism, malnutrition, cachexia, hepatic and renal involvement, chronic pancreatitis) in order to maintain the function of the other organs. After all, FG requires aggressive emergency therapy.

\section{Conclusion}

The cause of FG was not the dislocated stent (inserted in 2008 to drain a pancreatic pseudocyst). Immunodeficiency favored by alcoholism and malnutrition had led to bacterial multiplication and necrosis of the overlying skin. The radiologists did not detect the stent on former CT and perforation of the bowel wall was not confirmed during surgery. Nevertheless, a laparotomy and derivative colostomy was still required as the patient had rectal incontinence. Additionally, the resulting defects from necrosis could not have been properly cleaned as they would have continuously been contaminated. To avoid this continuous contamination, the decision of sigmoidal anus praeter was made.

It is, however, important to point out that the main problem for this patient was his severe clinical history. Overall, his alcoholism, chronic hepatitis, calcified chronic pancreatitis, decreased metabolism, immunodeficiency, cachexia, negative nitrogen balance, and neuropathy (Korsakoff's syndrome with sensory and motor dysfunction of the lumbar plexus and incontinence) were seen as very unfavorable.

In conclusion, FG spread to male genital organs presents therapeutic challenges; a well-organized and immediate multidisciplinary collaboration at the time of surgical debridement is required for optimal wound management and to sustainably lower the risk of retractile scarring.

\section{Disclosure}

The authors report no conflicts of interest in this work. 


\section{References}

1. Nakatani H, Hamada S, Okanoue T, et al. Fournier's gangrene in elderly patient: report of a case. J Med Invest. 2011;58(3-4):255-258.

2. Papachristodoulou AJ, Zografos GN, Papastratis G, et al. Fournier's gangrene: still highly lethal. Langenbecks Arch Chir. 1997;382(1): 15-18.

3. Ahmadnia H, Molaei M, Yaghoobi S, et al. New prognostic factors in Fournier's gangrene: a 10-year experience. Uro Today Int J. 2009; 2(4).

4. Fournier AJ. Gangrene foudroyante de la verge [Fulminating gangrene of the penis]. Sem Med. 1883;3:345. French.

5. Ferreira PC, Reis JC, Amarante JM, et al. Fournier's gangrene: a review of 43 reconstructive cases. Plast Reconstr Surg. 2007;119(1): 175-184.

6. Morpurgo E, Galandiuk S. Fournier's gangrene. Surg Clin North Am. 2002;82(6):1213-1224.

7. Pour SM. Use of negative pressure wound therapy with silver base dressing for necrotizing fasciitis. J Wound Ostomy Continence Nurs. 2011;38(4):449-452.

8. Kujath P, Kruger S, Bechtold H, Eckmann C. Diagnose und therapie der streptokokkenmyositis [Diagnosis and treatment of streptococcal myosistis]. Dtsch Med Wochenschr. 2002;127(47):2508-2510.
9. Marsman JW, Hoedemaker HP. Necrotizing fasciitis: fatal complication of migrated biliary stent. Australas Radiol. 1996;40(1):80-83.

10. Yılmazlar T, Işık Ö, Öztürk E, Özer A, Gülcü B, Ercan İ. Fournier's gangrene: review of 120 patients and predictors of mortality. Ulus Travma Acil Cerrahi Derg. 2014;20(5):333-337.

11. Wróblewska M, Kuzaka B, Borkowski T, Kuzaka P, Kawecki D, Radziszewski P. Fournier's gangrene - current concepts. Pol J Microbiol. 2014;63(3):267-273.

12. Pastore AL, Palleschi G, Ripoli A, et al. A multistep approach to manage Fournier's gangrene in a patient with unknown type II diabetes: surgery, hyperbaric oxygen, and vacuum-assisted closure therapy: a case report. J Med Case Rep. 2013;7:1.

13. Pais VM Jr, Santora T, Rukstalis DB. Fournier gangrene. Medscape. Available from: http://emedicine.medscape.com/article/2028899. overview. Accessed January 14, 2015.

14. Capelli-Schellpfeffer M, Gerber GS. The use of hyperbaric oxygen in urology. J Urol. 1999;162(3 Pt 1):647-654.

15. Grabe M, Bjerklund-Johansen TE, Botto H, et al. Guidelines on urological infections. Guidelines presented at: European Association of Urology; March 2011; Arnhem, the Netherlands.

16. Levett D, Bennett MH, Millar I. Adjunctive hyperbaric oxygen for necrotizing fasciitis. Cochrane Database Syst Rev. 2015;1:CD007937.
Clinical Interventions in Aging

\section{Publish your work in this journal}

Clinical Interventions in Aging is an international, peer-reviewed journal focusing on evidence-based reports on the value or lack thereof of treatments intended to prevent or delay the onset of maladaptive correlates of aging in human beings. This journal is indexed on PubMed Central, MedLine,

\section{Dovepress}

CAS, Scopus and the Elsevier Bibliographic databases. The manuscript management system is completely online and includes a very quick and fair peer-review system, which is all easy to use. Visit http://www.dovepress. com/testimonials.php to read real quotes from published authors. 